were investigated by lymphography and showed lymphatic abnormalities, but no one type of abnormality was present in all cases. It is interesting to note that abnormal lymphatics were detected in limbs in which there was no oedema, and this may indicate a generalized lymphatic abnormality varying in degree in different parts of the body.

P. A. Emerson ${ }^{8}$ has drawn attention to the association of yellow nails with lymphoedema and pleural effusions. P. A. Hurwitz and D. J. Pinals ${ }^{9}$ described two patients with pleural effusion and lymphoedema only. It is thought that an otherwise unexplained pleural effusion may occur in patients with chronic lymphoedema due to deficient lymphatic drainage of the pleural cavity. This may occur in association with yellow nails. Of the three cases of pleural effusion described by Emerson two had had recent attacks of bronchitis, after which they remained short of breath. One was subsequently shown to have a positive smear for tuberculosis, though the organism was never cultured and the patient did not respond to antituberculous therapy.

With the advance of lymphography abnormal lymphatics have been detected in patients with no sign of oedema, and it is suggested that oedema may develop after infection or trauma and give rise to an increased load on a deficient lymphatic drainage. The same may also be true in the pleural cavity, and certainly two of Emerson's patients had had an attack suggestive of recent infection. While lymphography from the arms and legs is now commonplace, visualizing the lymphatics draining the pleural cavity and many other regions of the body is either inadequate or impossible. But as techniques improve so may many diseases be shown to be due to lymphatic abnormalities, and the treatment of many conditions may be influenced by our greater understanding of this system.

\section{Lung Transplantation in Man}

It is now 16 years since the first mammalian lung was successfully transplanted experimentally. In dogs either the right or left lung may be removed and transplanted back into the same animal or another animal, anastomosing bronchus to bronchus, pulmonary artery to pulmonary artery, and atrial cuff containing the pulmonary veins to the left atrium. If the operation is performed within an hour ischaemic damage is unlikely to be severe. In fact, the lung is more resistant to ischaemic damage than the kidney. If both lungs are transplanted, or if one lung is transplanted and the other removed, there may be severe physiological disturbance. ${ }^{2}$ Thus, sudden increase in blood flow through the transplanted lung due to obstruction of the opposite pulmonary artery may produce acute pulmonary oedema, ${ }^{3}$ while total denervation of both lungs produces unusually slow and deep respiration owing to abolition of respiratory reflexes. In contrast to the kidney, where denervation affects function very little, an intact nerve supply to the lung is extremely important to health. Good results have been reported when no attempt has been made to anastomose the bronchial vessels or lymphatics.

I Juvenelle, A. A., Citret, C., Wiles, C. E., and Stewart, J. D., F. thor Surg., 1951, 21, 111 .

Brit. med. F., 1963, 1, 1302.

- Alican, F., and Hardy, J. D., f. Amer. med. Ass., 1963, 183, 849.

- Hardy, J. D., Eraslan, S., Dalion, M. L., Alican, F., and Turner, D. Ann. Surg., 1963, 157, 707.

Webb, W. R., Dalton, M. L., and Walker, G. R., f. Amer. med. Ass., 1963, 186, 1065.

- Magovern, G. J., and Yates, A. J., Ann. N.Y. Acad. Sci., 1964, 120, 710.

, White, J. J., et al., Canad. med. Ass. F., 1966, 94, 1199.
When a lung is transplanted from one animal to another it undergoes immune rejection, as a transplanted kidney does. This results in destruction of the lung in an untreated recipient after a few days. As in the kidney, so in the lung parenchyma, there is perivascular infiltration of mononuclear cells. In animals it has been shown that azathioprine (Imuran) is an effective immunosuppressive drug, and animals with transplanted lungs have survived for considerable periods after treatment with it." Three reports have been published of lung transplants in man. ${ }^{5-7}$ The recipients were dying from irreversible pulmonary disease, and the transplanted lungs were taken from cadavers. However, all three recipients died soon after transplantation $(18,8$, and 7 days after operation), and in two of the three cases there was severe bronchopneumonia in the transplant at post-mortem examination. In one case the bronchus of the transplanted lung was infarcted, possibly owing to inadequate bronchopulmonary arterial anastomoses. Though the transplanted lungs functioned satisfactorily in these cases, the clinical value of lung transplants has not yet been proved.

If one lung is transplanted in man the physiological disturbances are not severe, since the intact nerves of the other lung maintain the respiratory reflexes and the pulmonary arterial pressure need not be unduly increased. The major difficulty is infection. This may exist in the recipient's remaining lung, trachea, and bronchial stump, and in the lungs of cadaver donors, since it is unusual for a person to die with completely sterile lungs. Even if infection can be eliminated from these sites the transplanted lung in a patient treated with immunosuppressive drugs is likely to be highly susceptible to it. In fact, pulmonary infection has been a common and often fatal complication of renal transplantation in man owing to the immunosuppressive therapy. Successful transplantation of lungs may be difficult to achieve in man.

\section{Relief from Hardship}

Inflation hits hardest at those who cannot compete in the struggle to keep wages in line with prices. Doctors and their relatives are not alone in finding increasing difficulty in maintaining reasonable standards when the only income is from a pension or an annuity ; but the profession has a long tradition of looking after its own, and this tradition should be maintained. The Royal Medical Benevolent Fund reports that in 1965 there was a drop in income from subscribers and a revenue deficit of $£ 6,869$. The number of subscribers who died or retired in the year was 704 .

Many younger doctors may be unaware of the work done by the fund. In addition to assisting elderly doctors and families with grants and annuities the fund also helps the widows and young children of doctors who die before they have established themselves and thus secured adequate insurance and pensions. The Ladies Guild provides more personal service with visits and small gifts of clothing and other necessities.

Seven-year covenants signed by doctors enabled the fund to recover over $£ 5,000$ in income tax in 1965 without extra cost to the subscribers, and the Honorary Treasurer will give details of this scheme to anyone who asks. Doctors might also like to contribute to the gifts that are distributed each year at Christmas. Details are given in a letter from the Honorary Treasurer, Mr. G. H. Bateman, at page 1268 of this week's B.M.f. 